\title{
Lenalidomide and Chronic Lymphocytic Leukemia
}

\author{
Ana Pilar González-Rodríguez, ${ }^{1}$ Angel R. Payer, ${ }^{1}$ \\ Andrea Acebes-Huerta, ${ }^{2}$ Leticia Huergo-Zapico, ${ }^{2}$ Monica Villa-Alvarez, ${ }^{2}$ \\ Esther Gonzalez-García, ${ }^{3}$ and Segundo Gonzalez ${ }^{2}$ \\ ${ }^{1}$ Department of Hematology, Hospital Universitario Central de Asturias, C/Celestino Villamil s/n, 33006 Oviedo, Spain \\ ${ }^{2}$ Department of Functional Biology, IUOPA, Universidad de Oviedo, C/Julián Claveria s/n, 33006 Oviedo, Spain \\ ${ }^{3}$ Department of Hematology, Hospital de Cabueñes, C/Cabueñes s/n, 33394 Gijón, Spain
}

Correspondence should be addressed to Ana Pilar González-Rodríguez; anapilargonzalez@gmail.com

Received 28 May 2013; Accepted 7 August 2013

Academic Editor: Alessandro Isidori

Copyright @ 2013 Ana Pilar González-Rodríguez et al. This is an open access article distributed under the Creative Commons Attribution License, which permits unrestricted use, distribution, and reproduction in any medium, provided the original work is properly cited.

\begin{abstract}
Lenalidomide is an oral immunomodulatory drug used in multiple myeloma and myelodysplastic syndrome and most recently it has shown to be effective in the treatment of various lymphoproliferative disorders such as chronic lymphocytic leukemia (CLL) and non-Hodgkin lymphoma. The mechanism of action of lenalidomide varies depending on the pathology, and in the case of CLL, it appears to primarily act by restoring the damaged mechanisms of tumour immunosurveillance. This review discusses the potential mechanism of action and efficacy of lenalidomide, alone or in combination, in treatment of CLL and its toxic effects such as tumor lysis syndrome (TLS) and tumor flare reaction (TFR), that make its management different from other hematologic malignancies.
\end{abstract}

\section{Introduction on Chronic Lymphocytic Leukemia}

Chronic lymphocytic leukemia (CLL) is the most common form of leukemia in adults in Western countries. In our population, the crude rate is 8.99 per 100.000 populations per year and the age-adjusted rate is 3.47 per 100.000 populations per year. Overall survival at 5 and 10 years is ranged between $87 \%$ and $73 \%$ for low-risk patients and $29 \%$ and $16 \%$ for high risk [1]. In addition, the clinical course of the disease is very heterogeneous; some patients require treatment after a long time, while others rapidly progress with a few months of survival. Patients who relapse frequently show poor prognostic genetic characteristics, such as del (17p) or TP53, that confer them resistance to cytostatic drugs such as fludarabine $[2,3]$.

Microenvironment and immune system play a key role in the pathogenesis of CLL. Tissue microenvironment signals promote leukemic cell proliferation, survival, and resistance to chemotherapy. For instance, IL- 4 secreted by $\mathrm{T}$ cells induces the overexpression of antiapoptotic proteins, such as bcl-2, in leukemic cells [4]. Additionally, one of the key features of CLL is the development of a progressive immunodeficiency which is associated with an increased incidence of infections and secondary malignancies. In addition, numerous qualitative and quantitative alterations affecting all components of the immune system including $\mathrm{T}$ cells, NK cells, dendritic cells, and cytokine production have been described $[5,6]$.

A better understanding of the biology of the disease and tumor microenvironment has opened new ways for the development of immunotherapy-based treatments. The use of immunotherapy is of particular interest in this disease because the alteration of the immune system is further aggravated by the use of chemotherapeutic agents such as fludarabine and cyclophosphamide with rituximab (FCR) which are the current standards in frontline therapy. It is interesting to highlight that in a high percentage of patients the cause of death is related to immunodeficiency. Presumably, the activation of the immune system may ameliorate the immunodeficiency and repair the antileukemic immunity producing durable clinical responses. 


\section{Mechanism of Action of Lenalidomide}

Lenalidomide is an antineoplastic agent that exerts its antitumor action through various mechanisms such as activation of the immune system, inhibition of angiogenesis, and direct antineoplastic effects. The mechanisms of action may vary according to the disease, but there is growing evidence indicating that lenalidomide does not have a direct cytotoxic effect on CLL cells, but instead, it acts primarily by promoting and restoring the function of the immune system. Contrarily, changes in the serum concentrations of VEGF or in the density of the microvasculature in the bone of CLL patients who responded to lenalidomide treatment have not been found [7].

Functional immune reconstitution seems essential for the antileukemic activity of lenalidomide in CLL [8]. When lenalidomide is administered in cycles of 21 days, there is a rapid increase of the number of lymphocytes in the offtreatment week [9]. The stimulation of the immune system seems to be pleiotropic affecting different cells and functions. Lenalidomide causes an overexpression of costimulatory molecules in leukemic lymphocytes inducing an "activation phenotype" that restores the humoral immunity and the production of immunoglobulins [10]. It also improves the function of $\mathrm{T}$ cells and the ability of leukemic cells to form synapses with $\mathrm{T}$ lymphocytes [11]. There is also an increase of the number and the cytotoxic capacity of NK cells and a reduction of the number and suppressor activity of Treg cells [12].

2.1. Effects of Lenalidomide on Leukemic Cells. In contrast to normal B cells, leukemic cells are poor antigen presenting cells. This is due to the fact that leukemic cells have a reduced expression of costimulatory molecules such as CD80 and CD86 and they have a defect in the formation of immunological synapse with T cells. After lenalidomide treatment, there is an overexpression of costimulatory molecules and activation markers in leukemic B cells such as CD40, CD80, CD86, CD54, CD95 (Fas), DR5 and HLA-DR [9, 13]. Immune activation in CLL and the overexpression of costimulatory molecules may not only be responsible for the antineoplastic activity of lenalidomide, but also for the tumor flare syndrome (TFR) that affects some CLL-treated patients [7]. It has also been observed that lenalidomide restores the humoral immunity, since it induces the expression of CD154 (also known as CD40L) on T cells, which not only increases the sensitivity of leukemic cells to apoptosis, but it also activates normal B cells favoring the production of antibodies. Among them, there are some anti-tumor antibodies such as antiROR1 [14].

\subsection{Lenalidomide Activates Other Lymphocyte Subpopula-} tions. In CLL patients receiving lenalidomide as a first-line monotherapy, quantitative changes in lymphocyte subpopulations were observed after 21 days of treatment. There was a decrease in the number of CD19/CD5+ leukemic cells and an increase in the percentage of CD4 T cells, CD8 T cells, and NK cells [13]. It is worth mentioning that lenalidomide activates
CD8 T cells and NK cells that play a key role in the tumor surveillance.

Using lenalidomide monotherapy as first line, it was reported that the absolute number of lymphocytes decreased (mainly at the expense of leukemic lymphocytes) after three cycles of treatment. There was an increase of IFN- $\gamma$ produced by CD 8 T cells and Treg compared with their levels before the treatment [15]. The early increase of IFN- $\gamma$ produced by CD 8 $\mathrm{T}$ cells indicates that the cytotoxicity mediated by these cells may be involved in the mechanism of action of lenalidomide [15]. Nevertheless, the levels of T cells returned to normal values after 14 months of treatment. Similarly, there was an increase in the levels of CD4 T cells that produce IL-2, IFN$\gamma$, and TNF- $\alpha$ with respect to the baseline values and normal individuals. The elevation of CD4 T cells persisted for three months, returning to normal values at fourteen months [15].

Many of the immunomodulatory effects of lenalidomide occur via secretion of cytokines. In the study Ferrajoli et al., patients who respond to lenalidomide showed significant increases in the IL-2R, IL-6, and IL-10 levels [7] that may have a direct effect on immune cells. Likewise, Davies et al. demonstrated that lenalidomide is able (in vitro) to stimulate mononuclear cells in an IL-2-dependent manner, so they are able to kill myeloma cells [16]. This effect was abolished by the depletion of CD56+ cells suggesting that NK or NKT cells mediate this effect. Moreover, an increase of CD56+ cells in responding patients was observed, suggesting that the lenalidomide therapeutic activity may be mediated by the increase of the number and function of NK cells [16].

In vitro, lenalidomide stimulates $\mathrm{NK}$ cell activity at least through the production of IL-2 by $\mathrm{T}$ cells increasing the antibody-dependent cellular cytotoxicity (ADCC) mediated by these cells [17]. In vivo, an increase of $\mathrm{NK}$ cells in lenalidomide-treated patients has been observed; however, these cells showed a decrease in its activation level, having a concomitant enhancement of the cytotoxicity of CD8 T lymphocytes [18]. In addition to NK cells, lenalidomide also induces NKT cell expansion and stimulates its antitumor activity [19].

Overall, lenalidomide has a pleiotropic effect promoting several elements of the antitumor immune response. The role of each mechanism of action on the therapeutic activity of lenalidomide remains to be elucidated.

2.3. Mechanism of Action of Lenalidomide Associated with Rituximab. We have observed that in CLL, lenalidomide mainly acts promoting the proliferation and activation of NK cells ex vivo. However, the capacity of lenalidomide to promote the antileukemic activity of NK cells is limited. This is due to the fact that tumor cells are detected by NK cells through changes in their receptors ligand expression. However, leukemic cells of CLL patients express low levels of ligands of $\mathrm{NK}$ cell activating receptors, probably due to immune evasion mechanisms, being highly resistant to NK cell-mediated activation. To increase the cytotoxic activity against leukemia cells, it is necessary to favor the recognition of leukemia cells by NK cells. According to this idea, lenalidomide is an attractive agent for combination with rituximab. 
Rituximab allows the elimination of CD20+ leukemia cells through the receptor for IgG called CD16 from NK cells, by antibody-dependent cytotoxicity or ADCC. In vitro, it has been reported that lenalidomide decreases the leukemic cells CD20 expression, antagonizing the effect of rituximab [20], but this has not been confirmed [13]. In addition, we have found that the lenalidomide effect on CD20 expression on leukemic cells is variable, but independently, lenalidomide has a synergistic effect with rituximab. Following this idea, lenalidomide is an attractive agent to combine with other agents which favour the activity of NK cells.

\section{Lenalidomide Efficacy}

3.1. Use of Lenalidomide in Chronic Lymphocytic Leukemia. Lenalidomide, a derivative of thalidomide, is an immunomodulatory drug with significant activity in CLL. The efficacy of lenalidomide in monotherapy is comparable to other single cytotoxic agents used in this disease. In recent years, several phase II clinical trials have demonstrated the effectiveness of lenalidomide in CLL patients who relapsed or were refractory to previous treatments that included fludarabine; better results have been obtained when used as a first-line treatment $[7,9,21]$.

The first clinical trial demonstrating the clinical activity of lenalidomide in CLL is a randomized phase II study analysing 45 patients with relapsed or refractory disease [21]. In this study, lenalidomide was initiated at $25 \mathrm{mg}$ daily in 28 day cycles. After two cases of TLS, the starting dose was reduced to $5 \mathrm{mg}$, with escalations to a maximum of $25 \mathrm{mg}$. In heavily pretreated patients with CLL (51\% fludarabine refractory), ORR was $47 \%$ and complete remission (CR) was $9 \%$ [21]. In another phase II trial, in 44 patients using a more cautious dosing schedule (starting at $10 \mathrm{mg}$ daily and increasing $5 \mathrm{mg}$ every 28 days to a maximum of $25 \mathrm{mg}$ ), overall response decreased to $32 \%$ (CR 7\%), but side effects were significantly reduced [7]. In a phase I study using a low starting dose of $2.5 \mathrm{mg}$, and escalating $5 \mathrm{mg}$ each 28 days to 10 , 15 , and $20 \mathrm{mg}$, one-third of patients could not escalate beyond $2.5 \mathrm{mg}$, although those who reached $20 \mathrm{mg}$ did not suffer any dose-limiting toxicities [22]. This schedule with a low initial dose and further escalation has been adopted in subsequent clinical trials. In another study with a pulse dosing schedule of 21 days with 21 days off, global responses decreased to $16 \%$, but toxicity was similar [10].

Of considerable interest is its usefulness in patients with adverse features. It has been reported that in patients with high-risk cytogenetic features, the ORR was $31-38 \%$; in cases with nonmutated $\mathrm{IgH}$, it was $24 \%$; and in patients with fludarabine-refractory disease, it was $25-30 \%[7,23]$. Likewise, lenalidomide treatment, alone or in combination, became an effective alternative in these patients. In CLL patients with $\operatorname{del}(17 \mathrm{p})$ pretreated with different regimens, 8 who were treated with lenalidomide obtained an ORR of $38 \%$ (CR 13\%) and an overall survival (OS) of 11 months, which are better results than obtained with other combinations currently used in these patients [24]. The ORR improved up to $72 \%$ with lenalidomide monotherapy as first-line treatment with a prolonged followup of 47 months [25].
The overall response rate (ORR) of lenalidomide monotherapy as first-line therapy was $65 \%$ in elderly patients [26]. This study demonstrates that longer therapy and higher doses of lenalidomide are more effective to obtain responses. Nevertheless, the ORIGIN trial (NCT00910910), which has recently evaluated the use of lenalidomide treatment as an initial therapy for CLL patients of 65 or older, showed higher rates of death in patients treated with lenalidomide compared with those treated with chlorambucil (hazard ratio (HR) of 1.92). FDA has halted this study after determining that this treatment was unlikely to achieve an improved progressionfree survival in these older patients (primary objective).

Table 1 shows the response, survival rates, and adverse effects of several studies using different regimens of lenalidomide monotherapy as first-line or salvage treatment in relapsed CLL patients. Most of these trials begin with a low dose and then try to escalate to the target dose [22, 26-28].

3.2. Lenalidomide with Rituximab in Induction. The efficacy of lenalidomide may be increased with the addition of other agents such as rituximab. In several phase II clinical trials, it has been demonstrated that the addition of rituximab improved the response rates without increasing the toxicity. In a high percentage of patients, the number of leukemic cells declines within 8 days after the start of the treatment.

Badoux et al. reported that with this combination, relapsed or refractory CLL patients achieved an ORR of $66 \%$ (including 12\% CR) and an estimated 36-month survival of $71 \%$ [29]. In this study, lenalidomide was started with a continuous dose of $10 \mathrm{mg}$ on day 9 of cycle 1 and rituximab was added weekly $\left(375 \mathrm{mg} / \mathrm{m}^{2}\right)$ during the first cycle and then monthly (cycles 3 to 12). Although it cannot be directly compared with the outcomes obtained in the monotherapy studies, the encouraging ORR and sustained responses observed suggest an increasing benefit with the addition of rituximab, with less side effects and better tolerance. Furthermore, rituximab administered before lenalidomide could also act as a debulking agent reducing the rate and severity of TFR.

In another phase II clinical trial using lenalidomide and rituximab as the first-line therapy, overall responses were higher than $90 \%$. Moreover, this combination was safe with an acceptable toxicity profile. Even seven patients with (17p) deletion showed an ORR of 53\% (CR 13\%) [30]. Responses with this combination were also obtained in $71 \%$ of patients refractory to lenalidomide monotherapy [31].

The overall conclusion from these studies is that continuous treatment with this combination may probably provide accumulative benefits [32]. Thus, combination of lenalidomide with rituximab could offer an effective alternative for patients who relapsed after fludarabine-containing chemoimmunotherapy. Nevertheless, further studies must be implemented to obtain definite conclusions.

3.3. Other Combinations with Lenalidomide Induction. A current phase II study using lenalidomide in combination with ofatumumab demonstrates an acceptable toxicity profile [33], and similar outcomes are shown in several phase I trials 
TABLE 1: Lenalidomide monotherapy trials in patients with CLL.

\begin{tabular}{|c|c|c|c|c|c|c|}
\hline References & Regimen & No & Response & TLS & TFR & AE grade $3-4$ \\
\hline $\begin{array}{l}\text { Chanan Kh [21] } \\
2007 \\
\text { Phase II } \\
\text { Relapsed or refractory }\end{array}$ & $\begin{array}{l}\text { Lenalidomide } 5 \mathrm{mg} \text { per day } \\
\text { escalating up to } 25 \mathrm{mg} \text { per day }\end{array}$ & 45 & $\begin{array}{l}\text { ORR } 47 \% \\
\text { RC } 9 \% \\
\text { OS: NR } \\
\text { TFS: NR }\end{array}$ & $5 \%$ & $58 \%$ & $\begin{array}{c}\text { Neutropenia } 70 \% \\
\text { Thrombocytopenia } 45 \% \\
\text { Anemia } 18 \%\end{array}$ \\
\hline $\begin{array}{l}\text { Ferrajoli [7] } \\
2008 \\
\text { Phase II } \\
\text { Relapsed or refractory } \\
\end{array}$ & $\begin{array}{l}\text { Lenalidomide } 10 \mathrm{mg} \text { per day } \\
\text { escalating up to } 25 \mathrm{mg} / \text { day } \\
\text { (5 mg each } 28 \text { days }) \\
\text { Median } 10 \mathrm{mg}\end{array}$ & 44 & $\begin{array}{c}\text { ORR } 32 \% \\
\text { RC: } 7 \% \\
\text { OS 73\% at } 14 \text { months } \\
\text { TFS: NR }\end{array}$ & 0 & $12 \%$ & $\begin{array}{c}\text { Neutropenia } 41 \% \\
\text { Thrombocytopenia } 13 \% \\
\text { Anemia } 3 \%\end{array}$ \\
\hline $\begin{array}{l}\text { Chen }[9,25] \\
2011 \\
\text { Phase II } \\
\text { Untreated }\end{array}$ & $\begin{array}{l}\text { Lenalidomide } 2.5 \mathrm{mg} \text { per week } \\
\text { escalating up to } 10 \mathrm{mg} \text { or } \\
25 \mathrm{mg} \text { if no response } \\
\text { Median } 18 \text { cycles }(2-33)\end{array}$ & 25 & $\begin{array}{c}\text { ORR } 72 \% \\
\text { OS } 85.3 \% \\
\text { TFS } 68.8 \% \\
\text { (estimated at } 3 \text { years) }\end{array}$ & 0 & $88 \%$ & $\begin{array}{c}\text { Neutropenia } 76 \% \\
\text { Thrombocytopenia } 28 \% \\
\text { Anemia } 20 \%\end{array}$ \\
\hline $\begin{array}{l}\text { Aue } 2010[10] \\
\text { Phase II } \\
\text { Relapsed or refractory }\end{array}$ & $\begin{array}{l}\text { Lenalidomide pulses: } \\
10-20 \mathrm{mg} \text { for } 21 \text { days followed } \\
\text { by } 21 \text { days of rest ( } 4-8 \text { cycles) }\end{array}$ & 31 & $\begin{array}{c}\text { ORR } 16 \% \\
\text { RC } 0 \\
\text { PFS responding } 16 \mathrm{~m} \\
\text { versus } 6 \mathrm{~m}\end{array}$ & 0 & $53 \%$ & $\begin{array}{c}\text { Neutropenia } 56 \% \\
\text { Thrombocytopenia 30\% } \\
\text { Anemia } 15 \% \\
\text { Infection }\end{array}$ \\
\hline $\begin{array}{l}\text { Badoux [26] } \\
2011 \\
\text { Phase II } \\
\text { Elderly } \\
\text { Untreated } \\
\end{array}$ & $\begin{array}{l}\text { Lenalidomide } 5 \mathrm{mg} \text { monthly } \\
\text { increases to } 25 \mathrm{mg} \text { as tolerated } \\
\text { until disease progression or } \\
\text { unacceptable toxicity }\end{array}$ & $\begin{array}{c}60 \\
(>65 \text { years }) \\
\text { median } 71 \text { years }\end{array}$ & $\begin{array}{c}\text { ORR } 65 \% \\
\text { RC } 10 \% \\
\text { OS } 88 \% \\
\text { TFS } 60 \% \\
\text { (estimated at } 2 \text { years) } \\
\end{array}$ & 0 & $52 \%$ & $\begin{array}{c}\text { Neutropenia } 83 \% \\
\text { Thrombocytopenia } 47 \% \\
\text { Anemia }<1 \% \\
\text { Infection } 10 \%\end{array}$ \\
\hline $\begin{array}{l}\text { Lamanna [27] } \\
\text { Phase II } \\
\text { Treated and untreated }\end{array}$ & $\begin{array}{l}\text { Continuous low dose } 2.5 \mathrm{mg} \\
\text { to } 5 \mathrm{mg} \text { is increased if } \\
\text { progression (up to } 20 \mathrm{mg} \text { ) }\end{array}$ & 21 & & $7 \%$ & $47 \%$ & $\begin{array}{c}\text { Neutropenia } 52 \% \\
\text { Thrombocytopenia } 24 \%\end{array}$ \\
\hline $\begin{array}{l}\text { Wendter [28] } \\
\text { CLL-009 } \\
2011 \\
\text { Phase II } \\
\text { Relapsed or refractory }\end{array}$ & $\begin{array}{l}\text { Initial doses of } 5,10 \text {, or } 15 \mathrm{mg} \\
\text { escalating up to } 25 \mathrm{mg} \text { (dose } \\
\text { finding) }\end{array}$ & 60 & $\begin{array}{l}\text { ORR } 37 \% \\
\text { RC } 3.4 \%\end{array}$ & $3.4 \%$ & $10 \%$ & $\begin{array}{c}\text { Neutropenia } 41,7 \% \\
\text { Thrombocytopenia } 25 \% \\
\text { Anemia } 8.3 \%\end{array}$ \\
\hline $\begin{array}{l}\text { Wendter [22] } \\
2012 \\
\text { CLL-001 } \\
\text { Phase II } \\
\text { Treated }\end{array}$ & $\begin{array}{l}\text { Continuous cycles of } 28 \text { days } \\
\text { increasing from } 2.5 \mathrm{mg} \text { to } \\
25 \mathrm{mg}\end{array}$ & 52 & $\begin{array}{l}\text { ORR } 12 \% \\
\text { RC } 0 \% \\
\text { SD } 58 \%\end{array}$ & & $10 \%$ & $\begin{array}{c}\text { Neutropenia } 65 \% \\
\text { Thrombocytopenia } 33 \% \\
\text { Anemia } 10 \%\end{array}$ \\
\hline
\end{tabular}

TLS: tumor lysis syndrome; TFR: tumor flare reaction; AE: adverse effects; NR: not reported; ORR: overall response; CR: complete remission; OS: overall survival; SD: stable disease; TFS: treatment-free survival; m: months.

with other combinations (fludarabine, cyclophosphamide, and lenalidomide [34]; fludarabine, rituximab, and lenalidomide [35]; the same combination followed of maintenance with lenalidomide and rituximab [36] or in combination with bendamustine [37] or flavopiridol [38] or alemtuzumab). The tolerated dose of lenalidomide in these combinations is generally low (5-10 mg) and its efficacy is higher than in lenalidomide monotherapy. In fludarebine-based combinations even at the lowest dose level, dose-limiting toxicities occurred in most patients.

The preliminary results obtained from a phase II study with lenalidomide plus dexamethasone show a significant activity in previously untreated CLL patients. Moreover, it is generally well tolerated and reduces the incidence of side effects, such as TFR, enabling the escalation to higher dose of lenalidomide [39].
In another trial using lenalidomide consolidation after 6 cycles of pentostatin, cyclophosphamide, and rituximab (PCR-L), an increase in the response quality was observed and negative minimal residual disease (MRD) was shown in some cases. The treatment-free survival (TLS) was higher than a historical control (79\% versus $66 \%$ at 30 months) and the main toxicity observed was hematologic, but no cases of TLS or TFR were described [40]. An improved quality of the responses was also reported using lenalidomide as consolidation after FR or FCR treatment [41, 42]. Currently, lenalidomide is under evaluation for maintenance "Continuum Studio" and in patients with monoclonal B lymphocytosis.

Table 2 shows the different studies using lenalidomide in combination with other agents in CLL patients. 
TABLE 2: Trials using lenalidomide in combination in CLL patients.

\begin{tabular}{|c|c|c|c|c|c|c|}
\hline References & Regimen & No. & TLS \% & TFR \% & AE 3-4 (\%) & Response \\
\hline $\begin{array}{l}\text { Chanan Khan } \\
\text { [31] } \\
2006-7 \\
\text { Phase II } \\
\text { Relapsed or } \\
\text { refractory }\end{array}$ & $\begin{array}{l}\text { Lenalidomide } 10 \mathrm{mg} \text { per day escalated up } \\
\text { to } 5 \mathrm{mg} \text { each } 1-2 \text { week (max } 25 \mathrm{mg}) 21 \\
\text { days each } 28 \\
\text { Rituximab } 375 \mathrm{mg} / \mathrm{m}^{2} \text { days } 1,8 \text {, and } 15 \\
\text { (cycle } 1 \text { ) and days } 1 \text { and } 15 \text { (cycles } 2-6 \text { ) }\end{array}$ & 30 & 5 & 8 & $\begin{array}{c}\text { Neutropenia } 70 \% \\
\text { Thrombocytopenia } 45 \% \\
\text { Anemia } 18 \%\end{array}$ & $\begin{array}{l}\text { ORR } 57.7 \% \\
\text { RC } 18 \% \\
\text { TFS } 19.4 \text { months } \\
\text { ORR } 38 \% \text { (high } \\
\text { risk cytogenetic) }\end{array}$ \\
\hline $\begin{array}{l}\text { James } 2011[30] \\
\text { Phase II frontline } \\
\text { CLL Research } \\
\text { Consortium } \\
\text { CRC-014 }\end{array}$ & $\begin{array}{l}\text { Lenalidomide cycle } 1: 2.5 \mathrm{mg} \text {; cycles } 2-7 \text { : } \\
5 \mathrm{mg} \text { escalated up to } 10 \mathrm{mg} \text { (days } 1-21 \\
\text { each } 28 \text { ) } \\
\text { Rituximab: } 50 \mathrm{mg} / \mathrm{m}^{2} \text { day } 29 ; 325 \mathrm{mg} / \mathrm{m}^{2} \\
\left.\text { day } 31 ; 375 \mathrm{mg} / \mathrm{m}^{2} \text { day } 33 \text { (cycle } 1\right), \\
375 \mathrm{mg} / \mathrm{m}^{2} \text { weekly (cycle } 2 \text { ) and } \\
375 \mathrm{mg} / \mathrm{m}^{2} \text { day } 1 \text { (cycles } 3-7 \text { ) }\end{array}$ & 69 & & 1.4 & $\begin{array}{c}\text { Neutropenia } 49 \% \\
\text { Thrombocytopenia } 6 \% \\
\text { Anemia } 11 \%\end{array}$ & $\begin{array}{c}\text { ORR 95\% } \\
(<65 \mathrm{y}) \\
\text { ORR 78\% } \\
(>65 \mathrm{y}) \\
\text { RC } 20 \%(<65 \mathrm{y}) \\
\text { RC } 8 \%(>65 \mathrm{y}) \\
\text { TFS } 19-20 \mathrm{~m} \\
\end{array}$ \\
\hline $\begin{array}{l}\text { Badoux }[29,43] \\
\text { Phase II relapsed } \\
\text { or refractory }\end{array}$ & $\begin{array}{l}\text { Lenalidomide was started on day } 9 \text { at } \\
\text { cycle } 1 \text { and on day } 1 \text { of the cycles } 3-12 \text { : } \\
10 \mathrm{mg} \text { continuously } \\
\text { Rituximab } 375 \mathrm{mg} / \mathrm{m}^{2} \text { weekly during } \\
\text { cycle } 1 \text { and on day } 1 \text { cycles } 3 \text { to } 12\end{array}$ & 59 & 1.7 & 27 & $\begin{array}{c}\text { Neutropenia } 73 \% \\
\text { Thrombocytopenia } 34 \% \\
\text { Anemia } 15 \% \\
\text { Infection } 15 \%\end{array}$ & $\begin{array}{l}\text { ORR } 66 \% \\
\text { RC } 12 \% \\
\text { OS: } 71 \% \text { at } 36 \\
\text { months } \\
\text { TFS: } 17.4 \mathrm{~m}\end{array}$ \\
\hline $\begin{array}{l}\text { Veliz } 2009[32] \\
\text { Phase II } \\
\text { Relapsed or } \\
\text { progression } \\
\text { after rituximab } \\
\text { Heavily treated }\end{array}$ & $\begin{array}{l}\text { Lenalidomide cycle } 1: 2.5 \mathrm{mg} \text { (days } 1-7) \text {, } \\
5 \mathrm{mg} \text { (days } 8-15), 10 \mathrm{mg}(15-21 \text { days) } \\
\text { followed by } 7 \text { days of rest and then } 20 \mathrm{mg} \\
21 \text { each } 28 \text { days } \\
\text { Rituximab } 375 \mathrm{mg} / \mathrm{m}^{2} \text { weakly each } 4 \\
\text { weeks (day } 15 \text { ) }\end{array}$ & 10 & $\begin{array}{c}(\mathrm{RF}) \\
12\end{array}$ & 12 & Neutropenia $41 \%$ & $\begin{array}{l}\text { ORR } 30 \% \\
\text { RC } 0\end{array}$ \\
\hline $\begin{array}{l}\text { Chen } 2012 \text { [39] } \\
\text { Phase II } \\
\text { frontline }\end{array}$ & $\begin{array}{l}\text { Lenalidomide: } 5 \mathrm{mg} \text { per day escalated } \\
5 \mathrm{mg} \text { each } 28 \text { days ( } \max 25 \mathrm{mg} \text { ) } \\
\text { Dexamethasone: } 12 \mathrm{mg} \text { days } 1-4 ; 14,21, \\
\text { and } 28 \\
\text { Maximum } 18 \text { cycles }\end{array}$ & 18 & 0 & 5 & $\begin{array}{c}\text { Neutropenia } 53 \% \\
\text { Neutropenia febrile } 24 \% \\
\text { Thrombocytopenia } 12 \%\end{array}$ & $\begin{array}{l}\text { 0R } 59 \% \\
\text { RC } 1 \% \\
\text { RP } 53 \%\end{array}$ \\
\hline $\begin{array}{l}\text { Badoux [33] } \\
\text { Phase II } \\
\text { Relapsed or } \\
\text { refractory }\end{array}$ & $\begin{array}{l}\text { Lenalidomide } 10 \text { mg per day } \\
\text { Ofatumumab weekly }\end{array}$ & 16 & NR & 13 & $\begin{array}{l}\text { Neutropenia } 50 \% \\
\text { Anemia } 13 \%\end{array}$ & $\begin{array}{l}\text { ORR } 63 \% \\
\text { RC: } 13 \% \\
\text { OS: NR } \\
\text { TFS: NR }\end{array}$ \\
\hline $\begin{array}{l}\text { Ferrajoli [44] } \\
2012 \\
\text { Phase II } \\
\text { Relapsed or } \\
\text { refractory }\end{array}$ & $\begin{array}{l}\text { Lenalidomide } 10 \mathrm{mg} \text { day } 9 \text { continued for } \\
24 \text { months } \\
\text { Ofatumumab weekly for } 3 \text { weeks ( } 300 \mathrm{mg} \\
\text { week; } 1000 \text { mg week } 2 \text { and thereafter) } \\
\text { monthly (months 2-6); every other } \\
\text { month (months 7-24) }\end{array}$ & 36 & 0 & 0 & $\begin{array}{c}\text { Neutropenia } 47 \% \\
\text { Thrombocytopenia } 9 \% \\
\text { Anemia } 6 \%\end{array}$ & $\begin{array}{l}\text { ORR } 68 \% \\
\text { RC } 24 \%\end{array}$ \\
\hline $\begin{array}{l}\text { Blum [38] } \\
2011 \\
\text { Phase I } \\
\text { Relapsed or } \\
\text { refractory very } \\
\text { adverse }\end{array}$ & $\begin{array}{l}\text { Lenalidomide } 2.5 \mathrm{mg} \text { escalating up to } \\
25 \mathrm{mg} \text { days } 1-21 \\
\text { Flavopiridol } 30 \mathrm{mg} / \mathrm{m}^{2} \text { in bolus followed } \\
\text { of } 30-50 \mathrm{mg} / \mathrm{m}^{2} \text { days } 1,8 \text {, and } 15 \text { (cycle } 1 \text { ) } \\
\text { and then days } 3,10 \text { and } 17\end{array}$ & 30 & 3 & 7 & $\begin{array}{c}\text { Neutropenia } 47 \% \\
\text { Thrombocytopenia } 60 \% \\
\text { Anemia } 33 \%\end{array}$ & $\begin{array}{l}\text { ORR } 57 \% \\
\text { RC: } 0 \% \\
\text { OS } 7 \mathrm{~m} \\
\text { TFS } 23 \mathrm{~m}\end{array}$ \\
\hline $\begin{array}{l}\text { GIMEMA [34] } \\
\text { LLC } 606 \\
\text { Phase I } \\
\text { Relapsed or } \\
\text { refractory }\end{array}$ & $\begin{array}{l}\text { Lenalidomide } 2.5 \mathrm{mg} \text { escalating up to } \\
15 \mathrm{mg} \text { Cyclophosphamide Fludarabine }\end{array}$ & 9 & 0 & 11 & $\begin{array}{c}\text { Neutropenia transitory } \\
3-4 \text { in the majority of the } \\
\text { patients }\end{array}$ & $\begin{array}{l}\text { ORR } 67 \% \\
\text { RC 33\% } \\
\text { NR } \\
\text { NR }\end{array}$ \\
\hline $\begin{array}{l}\text { Egle [36] } \\
2011 \\
\text { Phase I/II } \\
\text { Frontline }\end{array}$ & $\begin{array}{l}\text { Lenalidomide } 2.5 \mathrm{mg} / \text { day (days } 7-21 \text { ) } \\
\text { escalating up to } 25 \mathrm{mg} \text { (day } 1-21) \\
\text { Fludarabine } 40 \mathrm{mg} / \mathrm{m}^{2} 1-3 \\
\text { Rituximab } 375 \mathrm{~m} / \mathrm{m}^{2} \text { day } 3 \text { cycle } 1 ; \\
500 \mathrm{mg} / \mathrm{m}^{2} \text { day } 1 \text { cycles } 2-6 \\
\text { Maintenance: } \\
\text { R: } 375 \mathrm{mg} / \mathrm{m}^{2} \text { cycles } 2,4 \text {, and } 6 \\
\text { Lenalidomide (maximum tolerated dose) }\end{array}$ & 45 & 0 & 0 & Neutropenia 88\% & $\begin{array}{l}\text { ORR } 87 \% \\
\text { RC } 49 \% \\
\text { NR } \\
\text { NR }\end{array}$ \\
\hline
\end{tabular}


TABLE 2: Continued.

\begin{tabular}{|c|c|c|c|c|c|c|}
\hline References & Regimen & No. & TLS \% & TFR $\%$ & AE 3-4 (\%) & Response \\
\hline $\begin{array}{l}\text { Flinn [45] } \\
2012 \\
\text { Phase I-II } \\
\text { Frontline }\end{array}$ & $\begin{array}{l}\left.\text { Rituximab } 375 \mathrm{mg} / \mathrm{m}^{2} \text { (cycle } 1\right) \\
500 \mathrm{mg} / \mathrm{m}^{2} \text { (cycle } 2-6 \text { ) } \\
\text { Fludarabine } 25 \mathrm{mg} / \mathrm{m}^{2} \text { (days } 1-3 \text { ) } \\
\text { Lenalidomide } 2.5-5 \mathrm{mg} \text { (days } 8-28) \\
6 \text { cycles }\end{array}$ & 51 & 6 & 0 & $\begin{array}{c}\text { Neutropenia } 47 \% \\
\text { Anemia } 14 \% \\
\text { Thrombocytopenia } 6 \\
\text { Rash } 14 \%\end{array}$ & $\begin{array}{c}\text { ORR: } 78 \% \\
\text { RC } 19 \% \\
\text { PFS } 71 \% \\
\text { OS } 88 \% \\
\text { (median FU } 21 \\
\text { months) }\end{array}$ \\
\hline $\begin{array}{l}\text { Brown [35] } \\
\text { Phase I } \\
\text { Frontline }\end{array}$ & $\begin{array}{l}\text { Fludarabine: } 25 \mathrm{mg} / \mathrm{m}^{2} \text { (days } 3-5 \text { ) } \\
\text { Rituximab: } 50 \mathrm{mg} / \mathrm{m}^{2} \text { day } 1 \text { and } \\
325 \mathrm{mg} / \mathrm{m}^{2} \text { day } 3 \\
\text { Lenalidomide: } 2.5 \mathrm{mg} \text { to alternate days ( } 21 \\
\text { each } 28 \text { days) } \\
\text { Followed by two cycles of consolidation } \\
\text { with lenalidomide }\end{array}$ & 9 & $1 / 9$ & $2 / 9$ & $\begin{array}{c}\text { Neutropenia } 66 \% \\
\text { Thrombocytopenia } 2 / 9 \\
\text { Allergy } \\
\text { Syndrome hand-foot }\end{array}$ & $\begin{array}{c}\text { ORR } 56 \% \\
\text { RC } 1 / 9\end{array}$ \\
\hline
\end{tabular}

TLS: tumor lysis syndrome; TFR: tumor flare reaction; AE: adverse effects; NR: not reported; ORR: overall response; CR: complete remission; OS: overall survival; TFS: treatment-free survival; y: year; m: month; RF: renal failure.

\section{Dose and Schedule of Lenalidomide in Patients with CLL}

The $25 \mathrm{mg}$ dose used in early studies was associated with excessive toxicity, and then subsequent trials started with lower doses (2.5-5 mg) progressively increased to $10-25 \mathrm{mg}$. In the majority of studies, the median of the tolerated dose was $10 \mathrm{mg}$. The use of lower doses may be associated with lower response rates, and dose escalations must be implemented to improve efficacy. Currently, daily doses used in trials range between 2.5 and $10 \mathrm{mg}$.

One of the open issues that needs further investigation is why a continuous dosing is more effective than 21 days schedules with a week off. The "recovery" of peripheral lymphocytosis observed using intermittent dosing of lenalidomide led to the current continuous daily dosing [9], since lymphocytosis recovery has not been noted in this regimen. Continuous dosing may prevent the leukemia cells recovery, inhibit the production of cytokines that promote the leukemia cells survival, and may also prevent the support of malignant cells by stromal cells [7]. Likewise, in patients with adverse prognostic features who were heavily pretreated and received 21 days of treatment with other 21 days off, the decrease of the toxicity, but also the efficacy, was observed [10].

As of yet, none of the clinical characteristics analyzed have allowed us to predict the response to lenalidomide treatment. Only one study reported that responders had a higher number of neutrophils than nonresponders [9].

\section{Side Effects}

The most common grade 3-4 adverse events of lenalidomide treatment were neutropenia, thrombocytopenia, and anemia. Tumor lysis syndrome (TLS), tumor flare reaction (TFR), and venous thromboembolism (VTE) will be discussed later. Skin rash, elevated liver enzymes, and phosphorus (P) and calcium (Ca) alterations are even more uncommon.
5.1. Tumor Flare Reaction (TFR). "Tumor flare reaction" (TFR) is a side effect unique of lenalidomide treatment in this disease that consists of the appearance of an increase in swelling of lymph nodes, spleen, and liver, with or without fever, erythema usually associated with local or generalized rash (maculopapular, erythematous, and nonpruritic), bone pain, and an increase in the number of lymphocytes. It is a self-limited and transient effect and can be managed with NSAIDs (ibuprofen $400-600 \mathrm{mg} / 6 \mathrm{~h}$ ) or a short course of steroids in severe cases. It is important to recognize it to avoid confusion with disease progression and improperly discontinue the treatment.

Using a starting dose of $25 \mathrm{mg}$, lenalidomide induced a significant response in CLL patients, associated with tumor lysis syndrome and TFR in a high percentage of cases [21]. These toxicities have been associated with a high starting dose and a rapid escalation of lenalidomide. With high doses of lenalidomide, it occurs within 6 days of treatment [7]; it is more common in the first few cycles of therapy and in previously untreated patients with a more robust immune system [9]. Although TFR is most common during the first cycle, repeated flare symptoms were also noted in $16 \%$ of the cycles upon resuming the lenalidomide treatment after the week off of each cycle, and it was observed as late as in cycle 28 [25]. It is also more common in patients with advanced stages of the disease and in patients with lymph nodes larger than $5 \mathrm{~cm}$ [7]. Contrarily, it has not been described in cases with low tumor mass, where lenalidomide is used as consolidation.

TFR was significantly reduced using low starting doses and using slower dose escalations [7]. In the study reported by Chanan Khan, using $20 \mathrm{mg}$ of prednisone in the first 5 days and $10 \mathrm{mg}$ another 5 days as prophylaxis, there was a decreasing in severity, but not in TFR incidence; nevertheless, none of the patients had to stop the treatment or reduce the dose. In this study, the onset of the syndrome was unrelated to the previous lymphocyte count or burden of the disease [21]. In a clinical trial reported by Chen et al., almost one-third of patients were treated with lower doses of prednisone (25$50 \mathrm{mg}$ for 5-10 days) and TFR was common, but mild [9]. 
The frequency of TFR also appears to be lower when lenalidomide is used in combination with rituximab. Using these drugs, TFR was also milder (grades 1 and 2) and occured during the first cycle of treatment [30]. The use of corticosteroids with lenalidomide plus dexamethasone is very interesting since it has significant activity in previously untreated CLL, is generally well tolerated and reduces dramatically the incidence of TFR symptoms. However, the use of other drugs with lenalidomide requires careful consideration of dosing and scheduling; for example, sequential treatment with ofatumumab and lenalidomide may be associated with a higher rate of TFR (57\%) than shown with concomitant therapy [46].

It has been suggested that TFR, a side effect observed only in CLL, may be secondary to the immune system activation. Accordingly, it has been reported that it is highly correlated with an overexpression of costimulatory molecules CD40, CD80, and CD86; and consequently, this "flare" may be due to an increased ability of leukemic B cells to present antigens and inducing an antitumoral response [47]. Accordingly, TFR has been correlated with clinical response [48]; however, this association has not been corroborated in all studies. On the other hand, it has also shown that progression-free survival in TFR patients is not better [23, 49].

The development of TFR has also been reported as a predictor of response [50]; however, it has not been corroborated in all studies.

5.2. Tumor Lysis Syndrome (TLS). Tumor lysis syndrome (TLS) is a specific adverse event more common in patients with high tumor burden or with regimens with a high starting dose of lenalidomide. TLS is a group of metabolic complications that appears after the lenalidomide treatment caused by the release of breakdown products by the lysis of leucemic cells. This syndrome includes hyperkalemia, hyperphosphatemia, hyperuricemia, hyperuricosuria, and hypocalcemia. It is mainly observed in the first 15 days of the treatment and it is more common in patients with bulky disease, moderate renal impairment, and elevated baseline uric acid levels. In some cases, it may progress to renal failure or arrhythmias that can be fatal [51].

Allopurinol (300 mg) is used for the prophylaxis of TLS, starting 3 days before the starting of the treatment and it is also used at the first cycle of each dose escalation (Celgene Corporation 2010). The dose should be adjusted in patients with renal insufficiency and patients should be adequately hydrated, drinking 8-10 glasses of water ( $240 \mathrm{~mL}$ each glass) a day for 14 days in the first cycle and for 14 days after each dose escalation (always taking into account the patient's cardiovascular status and the possibility of overloading volume). Additionally, uric acid, $\mathrm{P}, \mathrm{Ca}$, and creatinine levels must be monitored; and patients with previous renal failure requiring dialysis or having creatinine clearance lower than $60 \mathrm{~mL} / \mathrm{min}$ should be excluded.

5.3. Venous Thromboembolism (VTE). Venous thromboembolism occurs in 5\% of patients at 2-4 months after the starting lenalidomide treatment and can be avoided with appropriate prophylaxis. It has been suggested that lenalidomide may increase the risk of VTE due to endothelial cell damage caused by the presence of high TNF- $\alpha$ serum levels; aspirin may be useful in its prophylaxis [50]. Celgene has updated the prophylactic anticoagulation protocols in patients with CLL; these guidelines include the use of aspirin in patients with 0-1 risk factors for VTE and low molecular weight heparin ( $40 \mathrm{mg}$ of enoxaparin or equivalent) or oral anticoagulants (INR target of 2-3) in patients with 2 or more thrombosis risk factors.

\section{Conclusions}

Lenalidomide alone or in combination (mainly with rituximab) offered an effective therapeutic alternative for patients who relapsed after fludarabine-containing chemoimmunotherapy. Consequently, it is necessary to prospectively compare this combination with other commonly used salvage regimens. We should pay special attention to TFR and TLS in early cycles of treatment and it is important to recognize them to avoid confusion with disease progression and improperly suspend the treatment. Although clinical responses to therapy occurred early in this treatment regimen, patients who received a long-term therapy obtained an improvement in the quality of the response. Durable responses may be achieved, although it needs a long time and the treatment must be prolonged until progression and/or CR. Shorter treatments can be effective when lenalidomide is used in combination with other drugs. Combination with agents that act synergistically favouring the activity of the immune system and agents which do not produce myelosuppression is of particular interest.

\section{Acknowledgment}

This work was supported by the Spanish grants of Fondo de Investigaciones Sanitarias (Institute Carlos III) PI12/01280.

\section{References}

[1] A. P. González Rodríguez, E. González García et al., "Chronic lymphocytic leukemia: epidemiological study and comparison of MDACC and GIMENA pronostic indexes," Medicina Clínica, vol. 133, no. 5, pp. 161-6, 2009.

[2] M. Hallek, K. Fischer, G. Fingerle-Rowson et al., "Addition of rituximab to fludarabine and cyclophosphamide in patients with chronic lymphocytic leukaemia: a randomised, open-label, phase 3 trial," The Lancet, vol. 376, no. 9747, pp. 1164-1174, 2010.

[3] T. Robak, A. Dmoszynska, P. Solal-Céligny et al., "Rituximab plus fludarabine and cyclophosphamide prolongs progressionfree survival compared with fludarabine and cyclophosphamide alone in previously treated chronic lymphocytic leukemia," Journal of Clinical Oncology, vol. 28, no. 10, pp. 1756-1765, 2010.

[4] X. Mu, N. E. Kay, M. P. Gosland, and C. Darrell Jennings, "Analysis of blood T-cell cytokine expression in B-chronic lymphocytic leukaemia: evidence for increased levels of cytoplasmic IL-4 in resting and activated CD8 T cells," British Journal of Haematology, vol. 96, no. 4, pp. 733-735, 1997. 
[5] G. Görgün, T. A. W. Holderried, D. Zahrieh, D. Neuberg, and J. G. Gribben, "Chronic lymphocytic leukemia cells induce changes in gene expression of CD4 and CD8 T cells," Journal of Clinical Investigation, vol. 115, no. 7, pp. 1797-1805, 2005.

[6] A. P. Gonzalez-Rodriguez, J. Contesti, L. Huergo-Zapico et al., "Prognostic significance of CD8 and CD4 T cells in chronic lymphocytic leukemia," Leukemia and Lymphoma, vol. 51, no. 10, pp. 1829-1836, 2010.

[7] A. Ferrajoli, B.-N. Lee, E. J. Schlette et al., "Lenalidomide induces complete and partial remissions in patients with relapsed and refractory chronic lymphocytic leukemia," Blood, vol. 111, no. 11, pp. 5291-5297, 2008.

[8] X. C. Badoux, J. Reuben, B. Lee et al., "Lenalidomide therapy is associated with normalization of lymphocyte populations and increase in immunoglobulin levels in elderly patients with chronic lymphocytic leukemia," Haematologica, vol. 94, article S94, 2009.

[9] C. I. Chen, P. L. Bergsagel, H. Paul et al., "Single-agent lenalidomide in the treatment of previously untreated chronic lymphocytic leukemia," Journal of Clinical Oncology, vol. 29, no. 9, pp. 1175-1181, 2011.

[10] G. Aue, S. Soto, J. Valdez et al., "Phase II trial of pulse dosed lenalidomide in previously treated chronic lymphocytic leukemia," Blood, vol. 116, abstract 1383, 2010.

[11] A. G. Ramsay, A. J. Johnson, A. M. Lee et al., "Chronic lymphocytic leukemia $\mathrm{T}$ cells show impaired immunological synapse formation that can be reversed with an immunomodulating drug," Journal of Clinical Investigation, vol. 118, no. 7, pp. 24272437, 2008.

[12] C. Galustian, B. Meyer, M.-C. Labarthe et al., "The anti-cancer agents lenalidomide and pomalidomide inhibit the proliferation and function of T regulatory cells," Cancer Immunology, Immunotherapy, vol. 58, no. 7, pp. 1033-1045, 2009.

[13] D. F. James, J. R. Brown, L. Werner et al., "Phenotypic changes associated with acute reductions in leukemia cell counts in patients with chronic lymphocytic leukemia (CLL) receiving lenalidomide as initial therapy," Blood, vol. 116, abstract 59, 2010.

[14] R. Lapalombella, L. Andritsos, Q. Liu et al., "Lenalidomide treatment promotes CD154 expression on CLL cells and enhances production of antibodies by normal B cells through a PI3-kinase-dependent pathway," Blood, vol.115, no. 13, pp. 26192629, 2010.

[15] B.-N. Lee, H. Gao, E. N. Cohen et al., “Treatment with lenalidomide modulates T-cell immunophenotype and cytokine production in patients with chronic lymphocytic leukemia," Cancer, vol. 117, no. 17, pp. 3999-4008, 2011.

[16] F. E. Davies, N. Raje, T. Hideshima et al., "Thalidomide and immunomodulatory derivatives augment natural killer cell cytotoxicity in multiple myeloma," Blood, vol. 98, no. 1, pp. 210216, 2001.

[17] T. Hayashi, T. Hideshima, M. Akiyama et al., "Molecular mechanisms whereby immunomodulatory drugs activate natural killer cells: clinical application," British Journal of Haematology, vol. 128, no. 2, pp. 192-203, 2005.

[18] I. Idler, K. Giannopoulos, T. Zenz et al., "Lenalidomide treatment of chronic lymphocytic leukaemia patients reduces regulatory T cells and induces Th17 T helper cells," British Journal of Haematology, vol. 148, no. 6, pp. 948-950, 2010.

[19] D. H. Chang, N. Liu, V. Klimek et al., "Enhancement of ligand-dependent activation of human natural killer T cells by lenalidomide: therapeutic implications," Blood, vol. 108, no. 2, pp. 618-621, 2006.
[20] L. Lapalombella, "Lenalidomide down-regulates the CD20 antigen and antagonizes direct and antibody-dependent cellular cytotoxicity of rituximab on primary chronic lymphocytic leukemia cells," Blood, vol. 112, pp. 5180-5189, 2009.

[21] A. Chanan-Khan, K. C. Miller, L. Musial et al., "Clinical efficacy of lenalidomide in patients with relapsed or refractory chronic lymphocytic leukemia: results of a phase II study," Journal of Clinical Oncology, vol. 24, no. 34, pp. 5343-5349, 2006.

[22] C.-M. Wendtner, P. Hillmen, D. Mahadevan et al., "Final results of a multicenter phase 1 study of lenalidomide in patients with relapsed or refractory chronic lymphocytic leukemia," Leukemia and Lymphoma, vol. 53, no. 3, pp. 417-423, 2012.

[23] T. Sher, K. C. Miller, D. Lawrence et al., "Efficacy of lenalidomide in patients with chronic lymphocytic leukemia with high-risk cytogenetics," Leukemia and Lymphoma, vol. 51, no. 1, pp. 8588, 2010.

[24] X. Badoux, M. Keating, S. O’Brien et al., "Patients with relapsed CLL and 17p deletion by FISH have very poor survival outcomes," Blood, vol. 116, abstract 1248, 2009.

[25] C. Chen, H. Paul, T. Wang et al., "Long-term follow-up of a phase 2 study of single agent lenalidomide in previously untreated, symptomatic chronic lymphocytic leukemia (CLL), Blood, vol. 120, abstract 718, 2012.

[26] X. C. Badoux, M. J. Keating, S. Wen et al., "Lenalidomide as initial therapy of elderly patients with chronic lymphocytic leukemia," Blood, vol. 118, no. 13, pp. 3489-3498, 2011.

[27] N. Lamanna, M. L. Heaney, P. G. Maslak et al., "A new paradigm in CLL: minimizing toxicity by using the minimum effective dose (MED) of lenalidomide for older patients with CLL," Journal of Clinical Oncology, vol. 29, abstract 6609, 2012.

[28] C. M. Wendtner, G. Fraser, T. Aurran et al., "Interim results for the safety and efficacy of different lenalidomide starting dose regimens in subjects with relapsed or refractory chronic lymphocytic leukemia (CC-5013-CLL-009 Study)," Blood, vol. 120, abstract 642, 2011.

[29] X. C. Badoux, M. J. Keating, S. Wen et al., "Phase II study of lenalidomide and rituximab as salvage therapy for patients with relapsed or refractory chronic lymphocytic leukemia," Journal of Clinical Oncology, vol. 31, pp. 584-591, 2013.

[30] D. F. James, J. R. Brown, L. Werner et al., "Lenalidomide and rituximab for the initial treatment of patients with chronic lymphocytic leukemia (CLL) A Multicenter study of the CLL research consortium," Blood, vol. 118, abstract 291, 2011.

[31] A. Chanan-Khan, "Targeting the microenvironment: a new treatment paradigm for chronic lymphocytic leukaemia," Haematologica Reports, vol. 2, abstract 14, 2006.

[32] M. Veliz, R. Santana, J. E. Lancet et al., "Phase II study of lenalidomide in combination with Rituximab for patients with $\mathrm{CD} 5+/ \mathrm{CD} 20+$ hematologic malignancies who relapse or progress after rituximab: interim analysis," Blood, vol. 114, abstract 2376, 2009.

[33] X. Badoux, S. O’Brien, G. Wierda et al., "Combination of ofatumumab and lenalidomide in patients with relapsed chronic lymphocytic leukemia: initial results of a phase II trial," Blood, vol. 116, abstract 2464, 2010.

[34] F. R. Mauro, D. Armiento, S. Orlando et al., "Cyclophosphamide and Lenalidomide (FCL) for previously treated patients with chronic lymphocytic leukemia (CLL): results of dose-finding phase of the GIMEMA LLC606 Study," Blood, vol. 116, abstract $1377,2010$.

[35] J. R. Brown, J. Abramson, E. Hochberg et al., "A phase i study of lenalidomide in combination with fludarabine and rituximab 
in previously untreated CLL/SLL," Leukemia, vol. 24, no. 11, pp. 1972-1975, 2010.

[36] A. Egle, M. Steurer, T. Melchardt et al., "The REVLIRIT CLL5 AGMT study-a phase I/II trial combining fludarabine/rituximab with escalating doses of lenalidomide followed by rituximab/lenalidomide in untreated chronic lymphocytic leukemia (CLL): results of a planned interim analysis," Blood, vol. 114, abstract 3453, 2009.

[37] C. S. Ujjani, S. M. Karim, T. Goswami et al., "Bendamustine and lenalidomide in relapsed adn refractory chronic lymphocytic leukemia," in Proceedings of the 14th Biennial International Workshop on Chronic Lymphocytic Leukemia (iwCLL'11), vol. 11, S245, 2011.

[38] K. A. Blum, L. Wei, J. A. Jones et al., "Activity of combined flavopiridol and lenalidomide in patients with cytogenetically high risk chronic lymphocytic leukemia (CLL): updated results of a Phase I trial," Blood, vol. 118, abstract 3910, 2011.

[39] C. Chen, H. Paul, L. Del Rizzo et al., "An open-label phase 2 study of lenalidomide in combination with oral dexamethasone in the previously untreated, symptomatic patients with chronic lymphocytic leukemia (CLL)," Blood, vol. 120, abstract 2895, 2012.

[40] T. Shanafelt, H. Tun, C. Hanson et al., "Lenalidomide consolidation after first-line chemoimmunotherapy for patients with previously untreated CLL," Blood, vol. 116, abstract 1379, 2010.

[41] C. Ujjani, S. Jamshed, K. Fitzpatrick et al., "Lenalidomide following fludarabine and rituximab in untreated chronic lymphomcytic leukemia," Journal of Clinical Oncology, vol. 29, 2011.

[42] J. V. Pozadzides, M. J. Keating, W. G. Wierda et al., "Initial experience with lenalidomide as consolidation treatment in patients with chronic lymphocytic leukemia and residual disease after chemotherapy," Blood, vol. 118, abstract 3907, 2011.

[43] X. C. Badoux, M. J. Keating, S. O’Brien et al., "Final analysis of a Phase 2 study of lenalidomide and rituximab in patients with relapsed or refractory chronic lymphocytic leukemia," Blood, vol. 118, abstract 980, 2011.

[44] A. Ferrajoli, L. Falchi, S. O’Brien et al., "Combination of ofatumumab and lenalidomide in patients with relapsed chronic lymphocytic leukemia (CLL): results of a phase II trial," Blood, vol. 120, abstract 720, 2012.

[45] I. W. Flinn, R. S. Cooper, D. S. Thompson et al., "Fludarabine, Rituximab, and Lenalidomide in Previously Untreated Patients with Chronic Lymphocytic Leukemia (CLL): a Phase I/II Trial of the Sarah Cannon Research Institute," Blood, vol. 120, abstract 715, 2012.

[46] L. J. Costa, S. Fanning, J. Stephenson et al., "Phase 2 trial of intracycle sequential ofatumumab and lenalidomide for the treatment of relapsed and refractory chronic lymphocytic leukemia," Blood, vol. 120, abstract 3933, 2012.

[47] L. A. Andritsos, A. J. Johnson, G. Lozanski et al., "Higher doses of lenalidomide are associated with unacceptable toxicity including life-threatening tumor flare in patients with chronic lymphocytic leukemia," Journal of Clinical Oncology, vol. 26, no. 15, pp. 2519-2525, 2008.

[48] A. Chanan-Khan, K. C. Miller, D. Lawrence et al., "Tumor flare reaction associated with lenalidomide treatment in patients with chronic lymphocytic leukemia predicts clinical response," Cancer, vol. 117, no. 10, pp. 2127-2135, 2011.

[49] D. James, E. D. Avery, L. Zhang et al., "Lenalidomide administered for the initial treatment of chronic lymphocytic leukemia (CLL) patients: in vivo modulation of the leukemia cell surface phenotype and association with tumor flare reaction (TFR)," Blood, vol. 114, abstract 3440, 2009.

[50] G. Aue, J. Nelson Lozier, X. Tian et al., "Inflammation, $\mathrm{TNF} \alpha$ and endothelial dysfunction link lenalidomide to venous thrombosis in chronic lymphocytic leukemia," American Journal of Hematology, vol. 86, no. 10, pp. 835-840, 2011.

[51] L. A. Moutouh-de Parseval, L. Weiss, R. J. DeLap, R. D. Knight, and J. B. Zeldis, "Tumor lysis syndrome/tumor flare reaction in lenalidomide-treated chronic lymphocytic leukemia," Journal of Clinical Oncology, vol. 25, no. 31, p. 5047, 2007. 


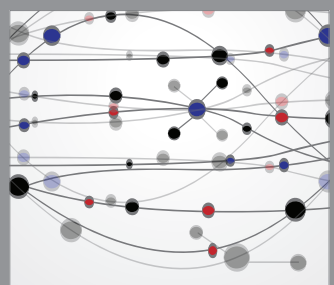

The Scientific World Journal
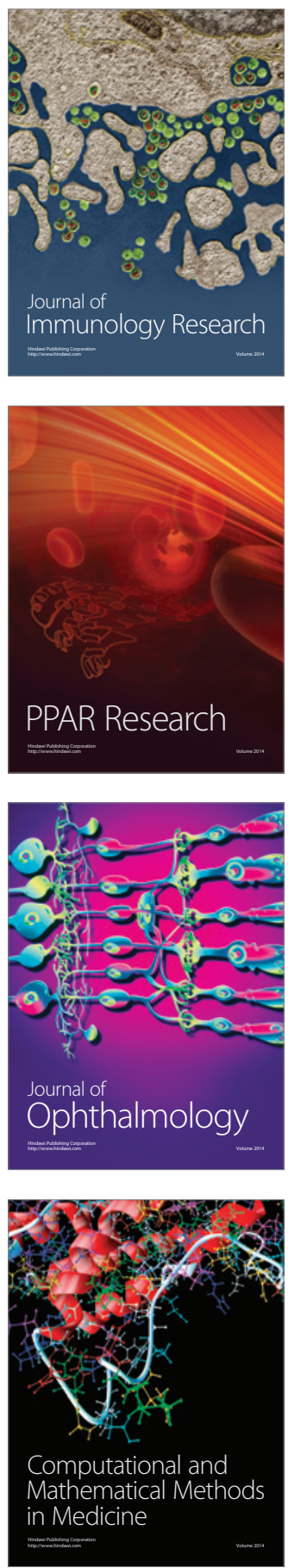

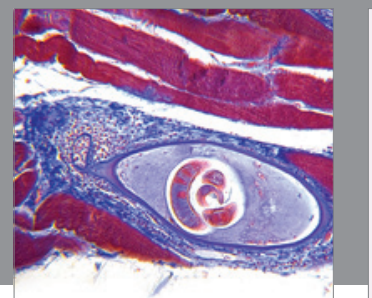

Gastroenterology

Research and Practice
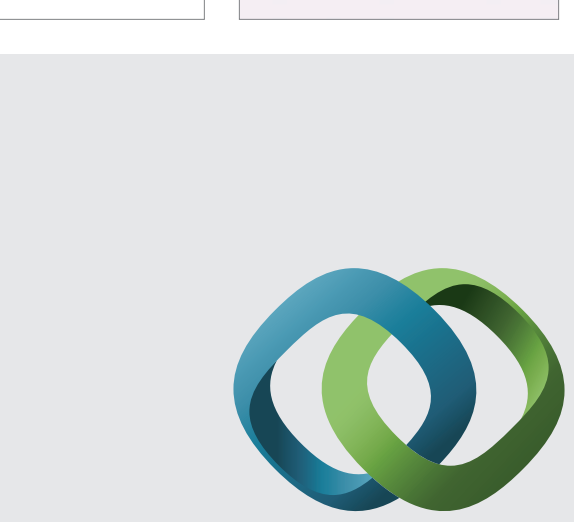

\section{Hindawi}

Submit your manuscripts at

http://www.hindawi.com
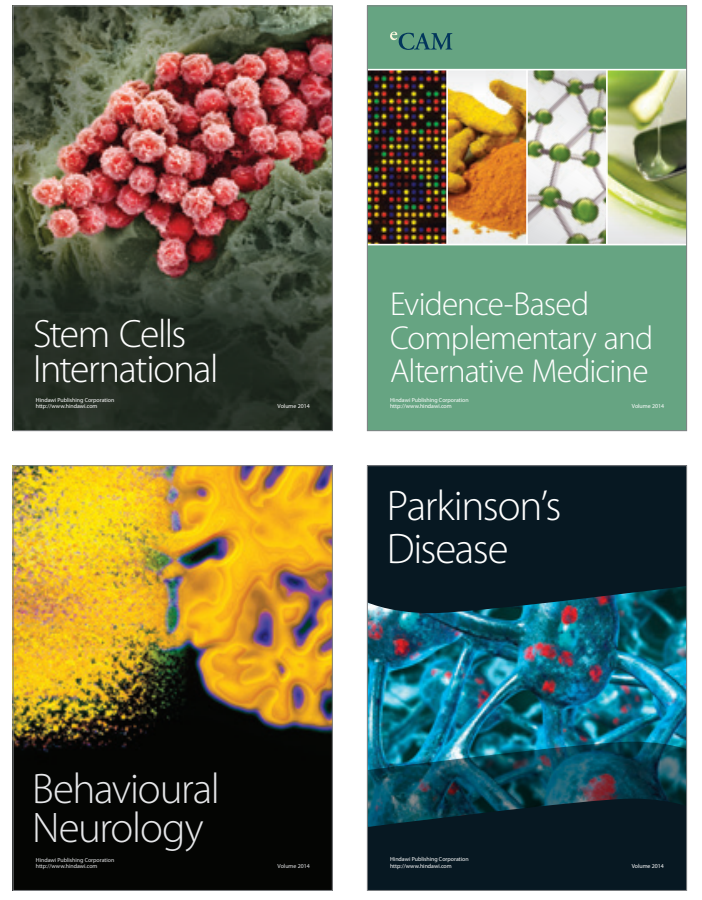
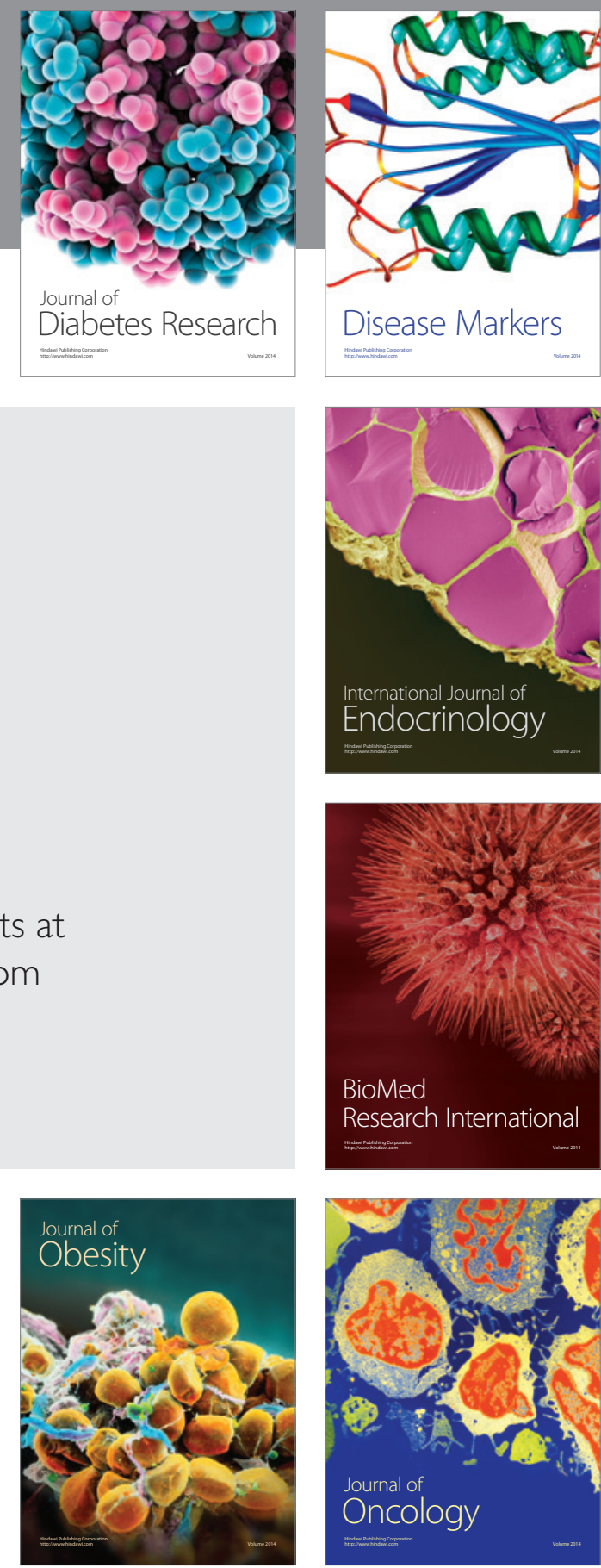

Disease Markers
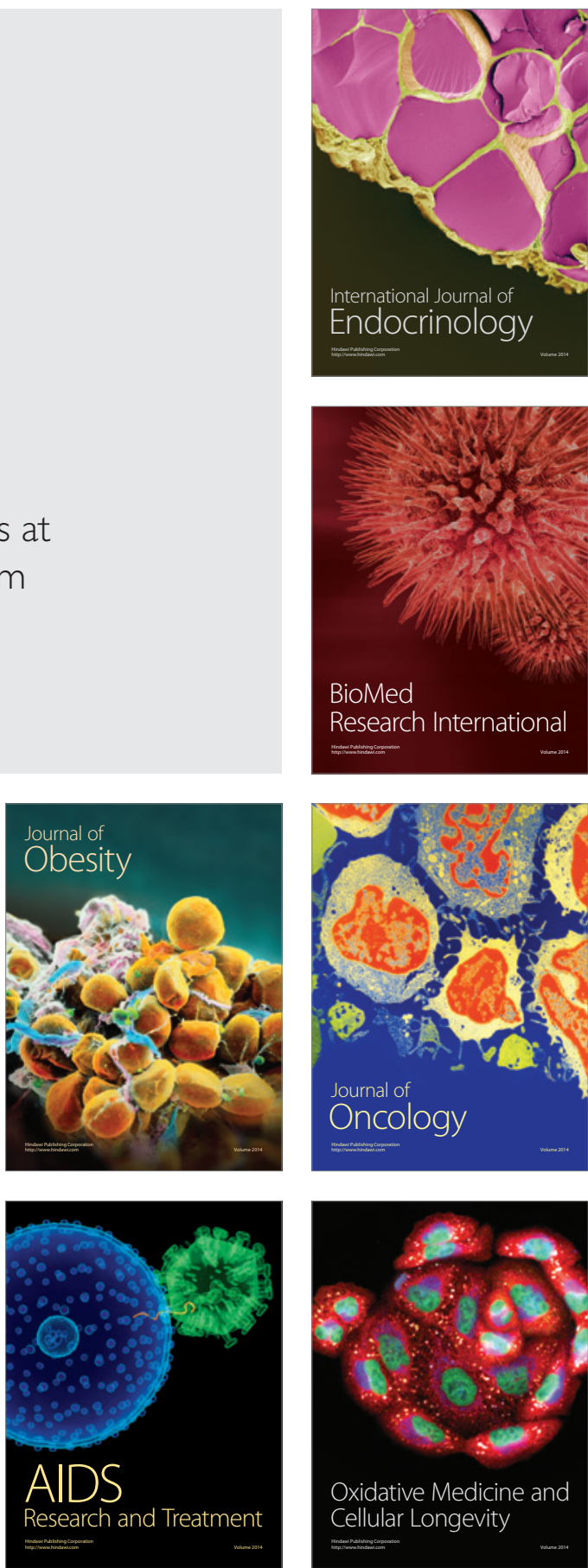